\title{
Realistic Simulation of Seasonal Variant Maples
}

\author{
Ning Zhou \\ Department of Computer Science and Technology \\ Tsinghua University \\ China \\ zhoun03@mails.tsinghua.edu.cn \\ Weiming Dong \\ Project ALICE \\ INRIA Lorraine, France \\ Weiming.Dong@loria.fr \\ Xing Mei \\ Sino French Lab in Computer Science Automation and Applied Mathematics \\ China \\ xmei@nlpr.ia.ac.cn
}

\begin{abstract}
This paper presents a biologically-motivated system of seasonal variant scenes generation for maples, which has a obvious leaf color transformation during the time. Given climate data and knowledge on environmental influence to maples, our system is able to simulate this seasonal leaf color transformation process. Our system consists of three steps: environment configuration, climate influence simulation and leaf texture acquisition. The first step decides the general color change timing of the maple tree based on its local environment. Then we make further adjustments to the timing determined in the last step taking into account the influence of climate in the specific case. In the last step, the texture maps of leaves are generated based on the pigment information. Our system is also able to simulate the seasonal color variance of other trees by adjusting related parameters.
\end{abstract}

\section{Introduction}

The appearance of computer-generated trees has been significantly improved with the help of recent advances in both modeling and rendering techniques. We are able to generate realistic pictures of natural scenes consisting different kinds of trees. However, an effective simulation of leaf color seasonal transformation is still not available. This transformation is caused by the change of pigment. As autumn comes, concentration of chlorophyll falls down because of the sunshine and temperature, so carotene is able to present its yellow color. Then anthocyanin is composed, and maple leaves change to red at the end[8]. This seasonal change happens on different scales: from the large scale

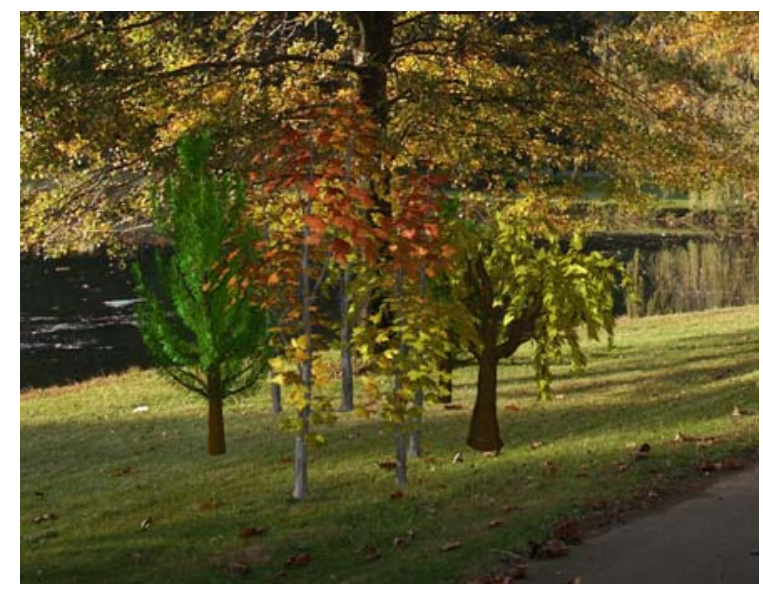

Figure 1. Rendering result of our system.

such as a forest to the small scale such as one piece of leaf. For a forest, the trees at different position change their leaf color at different time; For a tree, the leaves at different position change their color at different time; Even for a single leaf, different parts of it can have very different colors. Our goal is to make a realistic simulation of this beautiful process.

In this paper, we present a framework that can simulate the seasonal variant appearances of maples realistically. A forest consisting of both maples and other trees can also be rendered by our system. The appearance of each maple tree is determined by its surrounding environment and climate conditions. The color of the maple leaves ranges from greenish yellow to flaming orange to brilliant red between spring and later autumn. We use texture mapping to display the patterns of maple leaves. Various leaf patterns are used 
for a single maple tree.

We set several key points in the leaf color seasonal change process to divide it into three typical stages: from greenish yellow to flaming orange and then to brilliant red. We use the concentration of chlorophyll (for green), carotene (for yellow) and anthocyanin (for red) as main parameters to calculate the pixel colors of the leaf texture. Sunlight, temperature and precipitation are used as determinative parameters for texture choice of leaves. A colorful autumn is produced under the joint effect of abundant sunlight, low temperature and ample precipitation. They can accelerate chlorophyll decomposition and promote anthocyanin formation.

This paper is organized as following: Section 2 makes a review of previous works, and introduces some necessary knowledge from the biology domain. Section 3 describes our system in detail, and section 4 shows some sample results of our system. Finally, section 5 draws the conclusion and points out some potential directions for the future work.

\section{Related work}

\subsection{Previous work on simulation of plant- environment interaction}

Simulation of environment influence on plant is an interesting subject for both computer graphics and biology. Related works have been done on all the three scales mentioned in section 1. In the leaf scale, the inhomogeneous pigment distribution of leaf is taken into count to simulate seasonal change of leaf color. In the tree scale, one leaf is generally considered to be one color, and attentions are mainly paid to leaf arrangement. In the forest scale, even one whole tree can be considered to be only one color, and the research emphasizes on tree distribution. In this scale, to get higher performance, the differences among leaves are usually ignored, so our work emphasizes on tree and leaf scale.

Chiba et al.[3] presented a procedural method for simulation of both leaf arrangement and leaf seasonal color based on the estimation of the amount and direction of sunlight. Mochizuki et al.[11] simulated the autumn coloring process of both maple leaves and entire maple trees based on fractal model. Mech et al. [10] presented a L-system based framework to simulate interactions between plants and environment at plant architecture level. Deussen et al.[6][5] introduced a system for simulating and rendering large-scale plant ecosystems, performing both biologically-based plant distribution simulation and acceptable rendering efficiency.

However, most of the previous works focus only on the effect of a single environmental factor, such as [11] on temperature, [2] and [3] on sunlight. Few of them consider the joint influence of more than one factors at the same time.
In our system, we make an instructive try of considering several environmental factors under a unified framework.

\subsection{Biology explanation of maple seasonal color variance}

Though the complicated reactions during maple leaf color change have not been fully understood yet, we have sufficient knowledge of the role pigments and environment factors play in this process to make a realistic simulation. The leaf color is the joint result of three key pigments: chlorophyll, carotene, and anthocyanin. Chlorophyll is responsible for the green, which is the main color of leaves in most of the time. It is a key component for photosynthesis. It keeps on being decomposed and synthesized during the whole process of leaf growth, until the synthesis reaction stops when temperature is low. Comparing with chlorophyll, carotene, which expresses the yellow color, is more stable. In the autumn, it still exists when chlorophyll is almost disappeared. It helps with energy transfer. Anthocyanin is produced by reactions between sugar and some proteins. This reaction only happens when the concentration of sugar is quite high, and also requires light. It is responsible for the red-purple color of leaves, and can protect leaf from getting burned by the light.

Generally speaking, a brilliant autumn with colorful maples depends on not only abundant precipitation during the summer, but also bright weather and distinct daynight temperature difference during the autumn[8]. A wet summer ensures the composition of adequate chlorophyll, which act an important role in sugar production. Those sugar not only nourish the plant, but also will later decompose to anthocyanin resulting in the red hue of maple leaf. Bright weather and distinct temperature difference in the autumn will give plant the sign of stopping composing chlorophyll, so that the effect of other pigments will show out.

From physical point of view, leaf color is determined by light absorption of pigments, which is a function of wavelength. The wavelength range of visible light extends approximately from $380 \mathrm{~nm}$ to $770 \mathrm{~nm}$. Thus, the effective energy of a spectrum sensed by human eyes is

$$
V=\int_{380}^{770} P(\lambda) v(\lambda) d \lambda
$$

and the tristimulus values are

$$
X^{(k)}=\int_{380}^{770} R(\lambda) \bar{x}_{k}(\lambda) d \lambda, k=1,2,3,
$$

where $\lambda$ is the wavelength. Here we assume that there is no interplay among the color performances of different pigments. The absorption spectra of the three beforementioned pigments are shown in Figure 2[7]. In our system, we do not directly simulate the change in color space, 


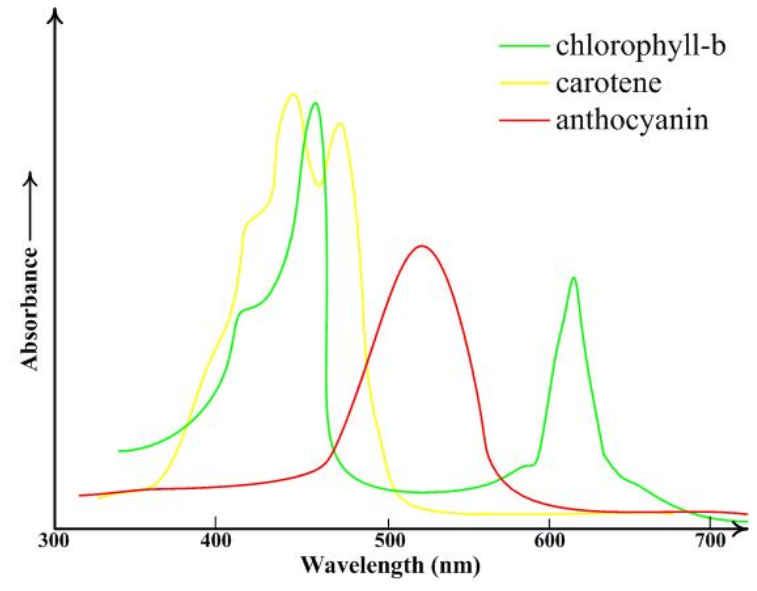

Figure 2. Absorbance of some important plant pigments for leaf color. Red, green and yellow lines represent the absorbance of anthocyanin, chlorophyll and carotene.

but compute the concentration change of the three key pigments instead.

\section{Simulation and visualization of maple sea- sonal color variance}

Our system simulates the leaf color seasonal change of maples emphasizing on tree scale. The whole system consists of three steps: (1) environment configuration, (2) climate influence simulation and (3) leaf texture acquisition. Step (1) decides the general color change timing of the maple tree based on its local environment. Step (2) makes further adjustments to the timing determined by step (1) considering the influence of climate in the specific case, and computes the pigment concentration for every leaf. Step (3) generates the leaf textures with the pigment concentration data. In this section, we describe the three steps in detail.

\subsection{Environment configuration}

Before consider the specific climate condition in a certain year, in this step, we first configure the normal climate parameters based on these basic environment conditions. Some certain environment conditions such as the latitude, longitude and altitude will generally decide the average climate parameters such as sunlight amount, precipitation and temperature, thus the approximate timing of maple leaf color change. Some previous work in biology domain has already provided some experience models on this subject, such as the ACLT (Autumn-Color-Logical-Timing) system[11]. In our system, we construct a look-up table for the relation between geography position and normal climate circumstances based on long term observation data[1]. Surrounding objects such as buildings and other trees will decrease the amount of light and rain and even heat the tree can receive, so in our system, we consider them as a weight coefficient $u$. After we determine the climate conditions including sunlight, temperature and precipitation, we simulate the concentration variation of the three key pigments.

The concentration of chlorophyll is in a dynamic balance of composition and decomposition. Its composition reaction requires more sunlight and higher temperature. So when autumn comes, the lack of sunlight and low temperature slow down the composition efficiency. As the concentration of chlorophyll falls down, carotene, which is more stable, is able to present its yellow color. At the same time, the low temperature also promotes the composition of anthocyanin, which expresses red in the acid chemical environment of maple leaves, so the leaves turn to red in the end [8]. A simple illustration of pigment concentration variance in this process is shown by Figure 4(a). Most relations between climate and pigment concentration can be approximated by a linear relation. The linear coefficient $\alpha$ is

$$
\alpha_{k}=\frac{u}{A_{k}} \int_{0}^{t} I_{k}(t) d t, k \in s, d, p
$$

where $u$ is the weight for surrounding environment, $A$ is the sum amount of the whole year in normal climate condition, and $I_{k}(t)$ is the weather circumstance at time $t$. The lower index $s$ is for sunlight, $d$ for temperature and $p$ for precipitation. Type of the pigment will be pointed out by the upper indices.

For chlorophyll, we assume $U_{c}(t)$ and $U_{d}(t)$ are its timedependent composition and decomposition speed. Its concentration at time $t$ can be given by

$$
c_{c h}(t)=\alpha_{d}^{c h} \alpha_{p}^{c h} \int_{0}^{t}\left(U_{c}^{c h}(t)-U_{d}^{c h}(t)\right) d t
$$

where $\alpha_{t}$ and $\alpha_{p}$ are influence factors of temperature and precipitation. Its composition and decomposition relies on not only sunlight but also other chemicals in the leaf, so we simulate them with the two-substrates model, which is a rectangular hyperbola as shown in Figure 3(a). For a twosubstrates reaction, suppose the utilization speeds of the two substrates are equivalent, then the two-substrates model can be described by

$$
U=\frac{k^{\prime} X_{1} X_{2}}{1+L_{1} X_{1}+L_{2} X_{2}+L_{12} X_{1} X_{2}}
$$

$U$ is the reaction speed. $k^{\prime}, L_{1}, L_{2}$ and $L_{12}$ are constants. $X_{1}$ and $X_{2}$ are the concentration of the two substrates. To simplify the model, we consider one of the substrates as constant. Then Equation 5 can be written in the form

$$
U=\frac{k X}{K+X}
$$




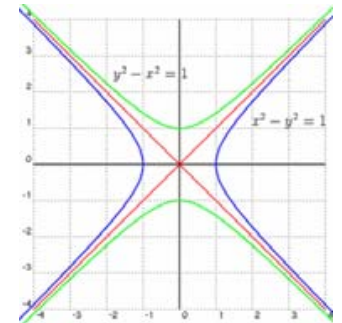

(a) The rectangular hyper- (b) The switch-off threshold response bola model

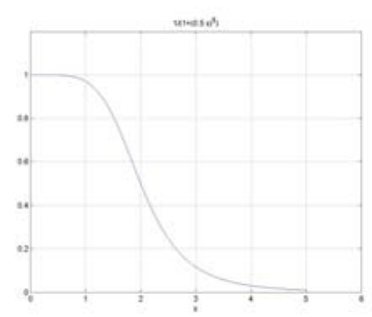
curve

\section{Figure 3. Mathematic models for pigment simulation.}

where $U$ is the reaction speed, $k$ and $K$ are constants, $X$ is the concentration of substrate. Equation 6 is a common-used rectangular hyperbola model: MichaelisMenten model[12]. To reveal the influence of sunlight, we let constant $k=\alpha I$ ( $I$ is light flux density, and $\alpha$ is the utilization efficiency constant)

For anthocyanin, temperature has a threshold effect to it: only when temperature is low enough will the reaction of anthocyanin composition start. Thus we use the switch-off threshold model to simulate this process. It corresponds to reactions which only happen when the value of influence factors reaches a certain level. So the concentration of anthocyanin at time $t$ is

$$
c_{a n}(t)=\alpha_{s}^{a n} \alpha_{p}^{a n} \int_{0}^{t} U^{a n}(t) d t
$$

where the reaction speed $U$ is

$$
\frac{U}{k}=\frac{1}{1+\left(X / X_{c}\right)^{n}}
$$

$k$ is a constant. $X$ is the value of influence factor, which is the temperature at time $t$ in our system. $X_{c}$ is the $X$ value corresponding to half maximum response. $n$ is a positive integer. The larger $n$ is, the more obvious the threshold effect is. In our system, we let $n=5$. The response curve is shown in Figure 3(b).

Concentration of carotene is generally considered as constant. All the before-mentioned models use empirical parameters obtained from previous observation data.

\subsection{Climate influence simulation}

A very accurate simulation requires massive climate data and chemical measurement data of the three main pigments, which are difficult to acquire. Therefore, in our system, based on limited observation data of several key stages, a standard process under normal climate conditions (Figure $4(\mathrm{a}))$ is generated by interpolation.

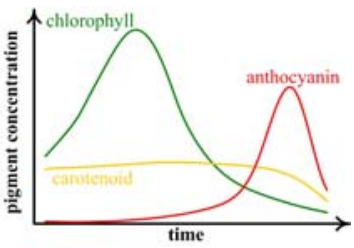

(a) Normal climate conditions

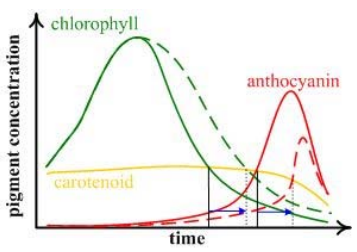

(c) Influence of warm autumn

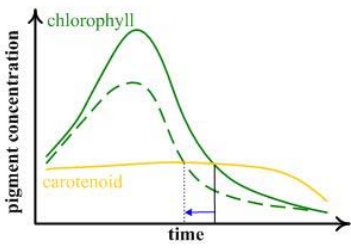

(b) Influence of precipitation deficiency

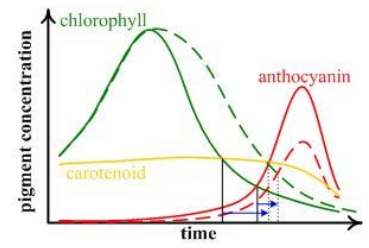

(d) Influence of sunlight deficiency
Figure 4. Influence of climate to pigment concentration. Figure 4(a) shows the pigment concentration under normal environment conditions. The dashed lines in Figure $4(b)$ to $4(d)$ indicate the concentration changes under abnormal climate conditions. Red, green and yellow lines represent the absorbance of anthocyanin, chlorophyll and carotene. The arrows show the change of color transformation timing.

In a certain case, the climate conditions have some differences from the normal case. These difference will influent the leaf color changing timing. In Figure 4, we analyze three typical cases of abnormal climate. Figure 4(b) is precipitation deficiency. Precipitation affects the production efficiency. Its deficiency causes a decrease of chlorophyll concentration, so the carotene is able to express its color earlier. The yellow stage starts earlier. Figure 4(c) is warm autumn. Temperature decides when the plant stops composition of chlorophyll, and starts to produce anthocyanin from sugar, so a warm autumn results in a lack of anthocyanin, and delays the red stage. Figure 4(d) is sunlight deficiency. Sunlight plays an important role in the photosynthesis, in which chlorophyll is consumed and energy is produced, so that deficient sunlight will retain chlorophyll and extend the green stage. In section 4 we show the corresponding rendering results of these climate conditions in Figure 7.

\subsection{Leaf texture acquisition}

According to botany knowledge, the color change process can be divided into several stages, from green to dark red. Though appearance differences exist among the leaves 


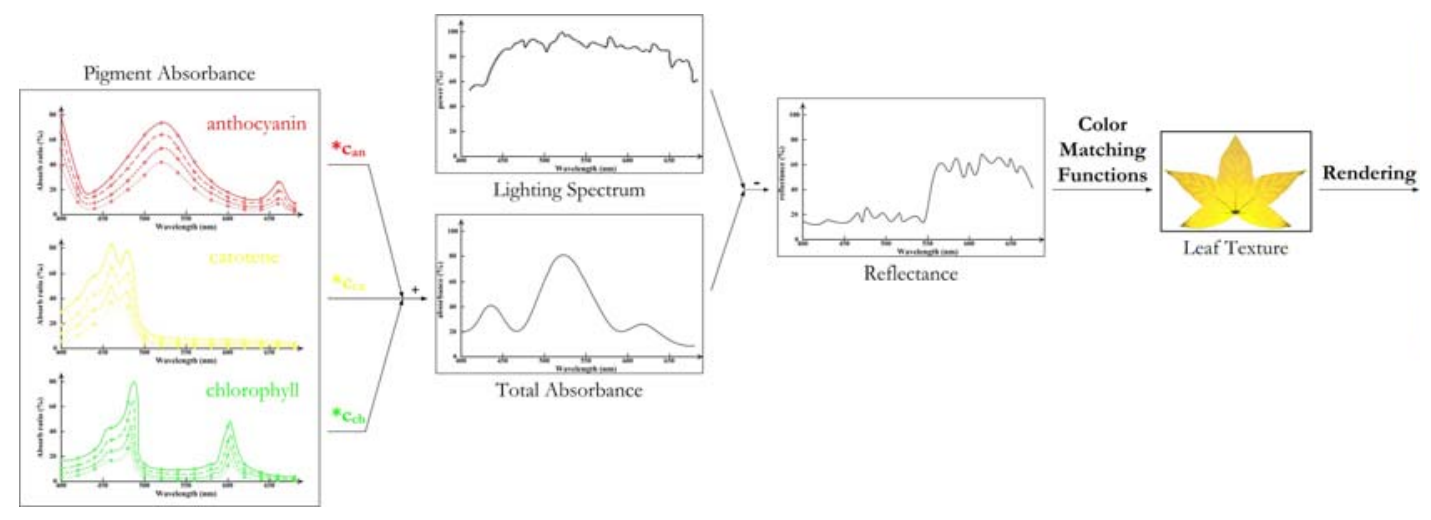

Figure 5. The illustration of leaf texture acquisition method.

even in the same stage, usually several sample textures obtained from photos of real leaves are used to make an index of all the stages. This method is simple and fast, but it decreases the diversity of leaves in the same stage, and results artifact sudden change between leaves of different stages. More accurate simulation can be made based on biological or mathematic models. Though this method requires more precomputation and storage space than the former one, it can achieve higher diversity. In this paper, we design a joint method of measurement and algorithmic to take the advantage of the both.

The pipeline of our leaf texture acquisition method is shown in Figure 5. We use the concentration of chlorophyll $c_{c h}$, carotene $c_{c a}$ and anthocyanin $c_{a n}$ as the input data to calculate the leaf color. Calculating the color of pigments mixture requires a subtractive color system rather than the common additive system. In our system, we directly subtract the pigment absorbance from lighting in the spectrum domain, and then use color matching functions to transform the reflectance spectrum to the color space we want. The calculations in spectrum domain are treated as a linear combination. According to Beer's law [9], the absorption spectrum of a pigment approximately follows a linear relationship with its concentration: $A_{c}(\lambda) \approx c \cdot A(\lambda)$. The basic absorption $A_{0}$ and transmission $T_{0}$ of leaf tissue, which vary among different plant species, are approximately by measure data from several typical leaf samples[4]. Thus the reflectance of the leaf is

$$
R(\lambda)=L(\lambda)-T_{0}(\lambda)-A_{0}(\lambda)-\sum_{p \in a n, c a, c h} c_{p} A_{p}(\lambda)
$$

And then, the leaf color can be calculated by Equation 1 and 2. A basic map containing spacial variance information such as the venation pattern is used to improve the final results.

\section{Result}

We use our system to simulate the seasonal color variance of maple trees. On a PC with P4 3.2GHz CPU, $1 \mathrm{~GB}$ RAM and a Geforce 7800 GT graphics card, the computation time for rendering a maple tree with 150 leaves is around 25 seconds. The performance is generally determined by the amount of leaves and observation step of climate data. Figure 1 shows the rendering result of our system. Figure 6 shows the leaf color change of a maple tree in the autumn as time passes.

Figure 7 shows the influence of different climate conditions. The four figures are the same scene in the same season. The scene consists not only maples, but also pine, which does not have the seasonal color change, and willow, which only change from green to yellow. Figure 7(a) is the simulation result with normal climate conditions. The maples start to turn to red, and the willow is yellow. Figure 7(b) to7(d) show the simulation results with precipitation deficiency, warm autumn, and sunlight deficiency. In Figure 7(b), precipitation deficiency causes a decrease of chlorophyll concentration, so the green stage ends earlier. More leaves of the maples are yellow and red. In Figure 7(c), warm autumn postpones the composition of anthocyanin, hence delays the red stage. In Figure 7(d), sunlight deficiency retains chlorophyll, so it inhibits the color express of carotene.

\section{Conclusion}

This paper introduces a system to simulate the seasonal color change of maple leaves. Based on the MichaelisMenten model and the threshold response model, concentration change precesses of three key pigments chlorophyll, carotene and anthocyanin are simulated. Parameters are estimated based on local environment conditions. Particular texture is generated for every leaf to achieve high diversity 


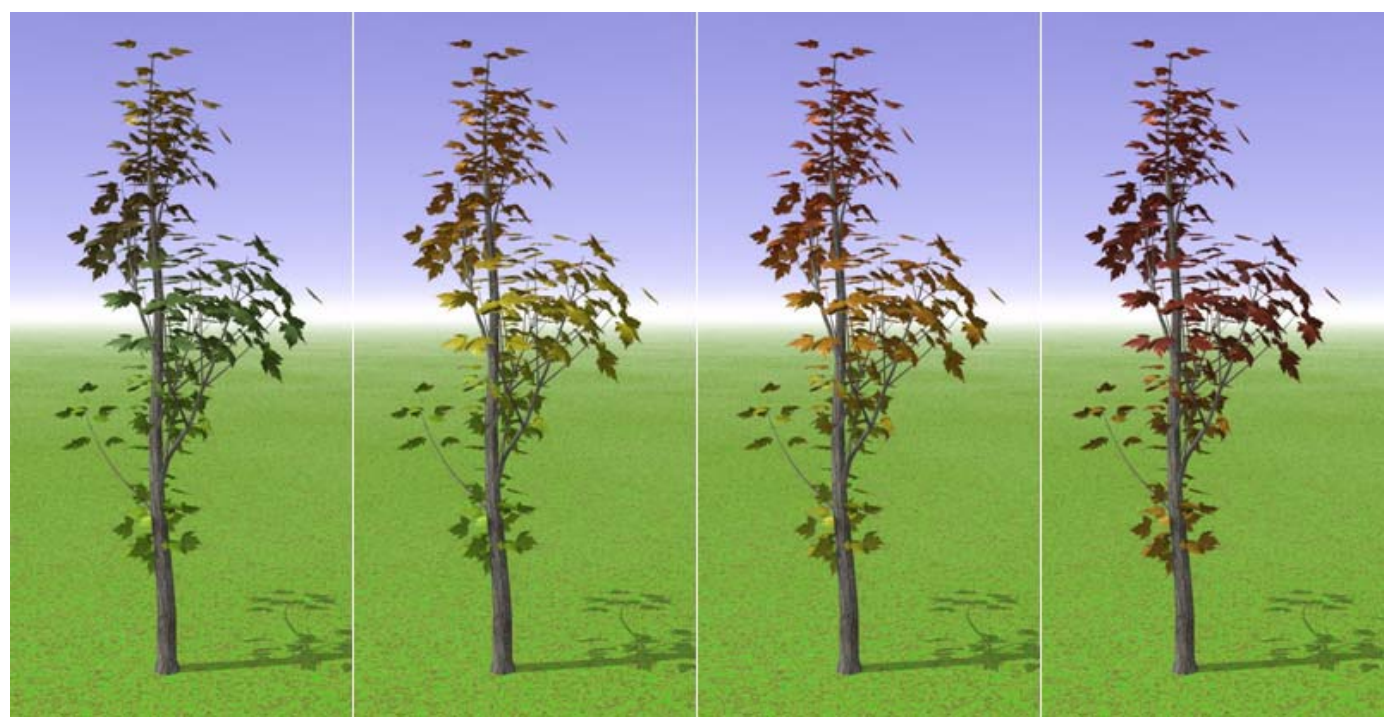

Figure 6. The sample result of our system.

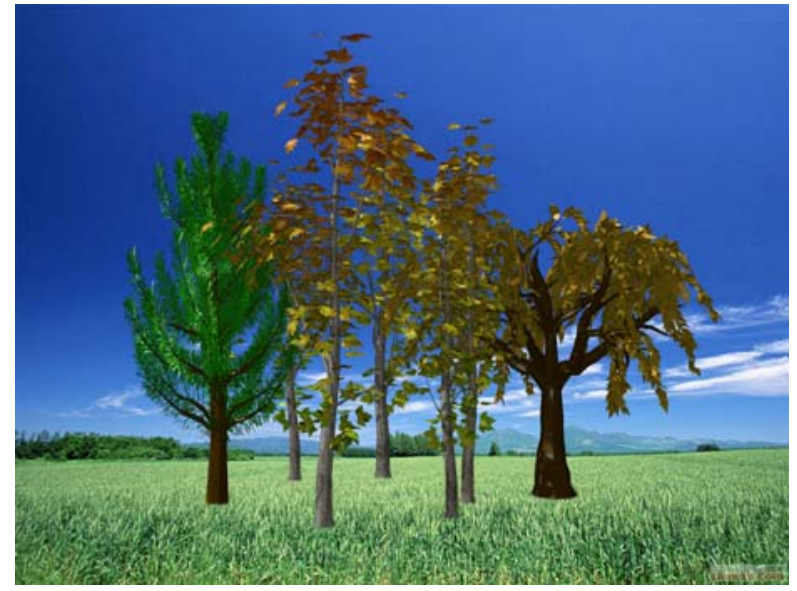

(a) Normal climate conditions

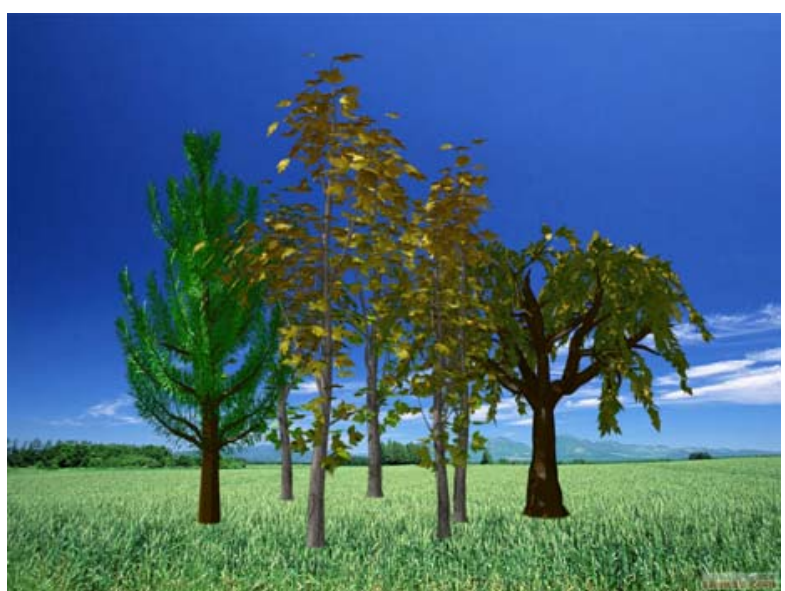

(c) Influence of warmer autumn

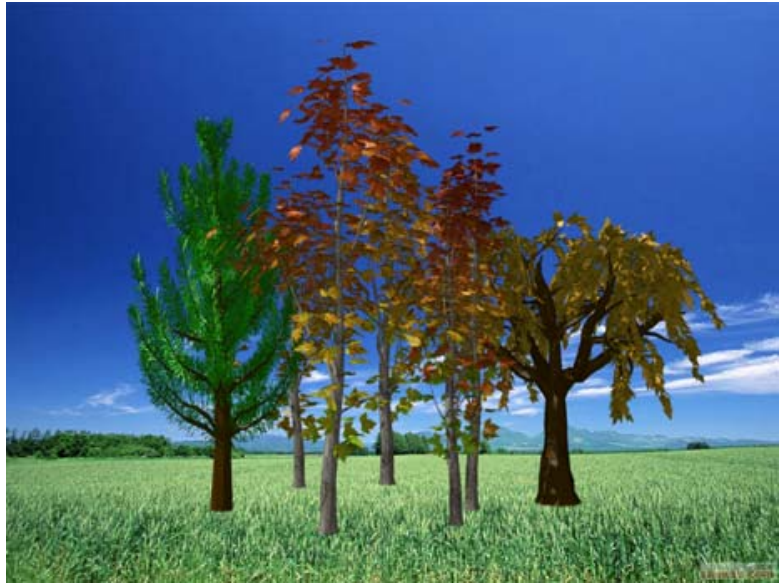

(b) Influence of precipitation deficiency

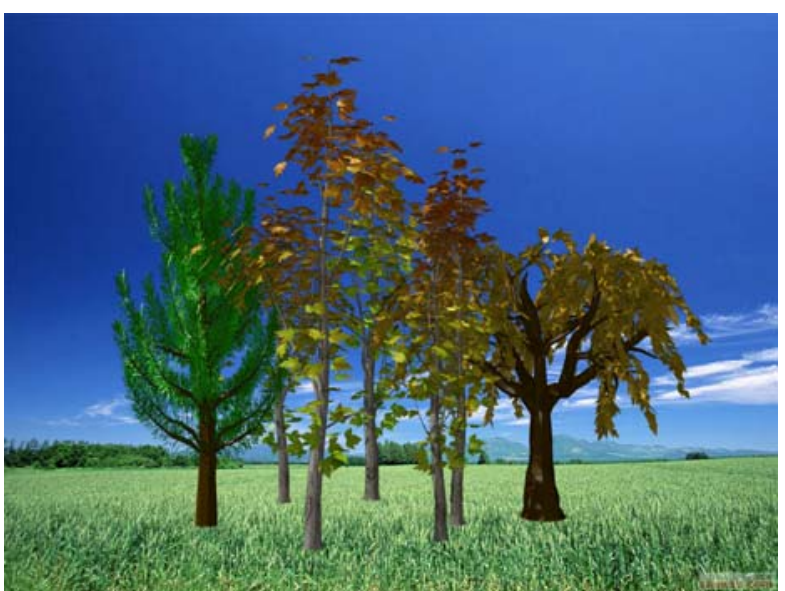

(d) Influence of sunlight deficiency

Figure 7. Simulation results corresponding to the influences of climate conditions in Figure 4. 
and natural appearance in the rendering result. Furthermore, given future climate data, Our system is able to predict the color transformation timing of maples. Influence of abnormal climate can also be simulated.

For future work, simulating the shape change of leaf during the growth is an interesting direction.

\section{6 acknowledgements}

The authors would like to thank Professor Xiaopeng Zhang for providing the 3D models of maple trees. Thanks to Professor Norishige Chiba of Iwate University for the useful information. This work is sponsored by the Sino French Lab in Computer Science, Automation and Applied Mathematics (LIAMA).

\section{References}

[1] http://www.ncdc.noaa.gov/oa/wmo/wdcamet.html.

[2] M. Braitmaier, J. Diepstraten, and T. Ertl. Real-time rendering of seasonal influenced trees. In P. Lever, editor, Procceedings of Theory and Practice of Computer Graphics 2004, pages 152-159. Eurographics UK, 2004.

[3] N. Chiba, K. Ohshida, K. Muraoka, and N. Saito. Visual simulation of leaf arrangement and autumn colours. The Journal of Visualization and Computer Animation, 7, 1996.

[4] T. P. Dawson, P. J. Curran, and S. E. Plummer. Liberty modeling the effects of leaf biochemical concentration on reflectance spectra. Remote Sensing of Environment, 65:5060, 1998.

[5] O. Deussen, C. Colditz, M. Stamminger, and G. Drettakis. Interactive visualization of complex plant ecosystems. In IEEE Visualization 2002, pages 219-226, 2002.

[6] O. Deussen, P. Hanrahan, B. Lintermann, R. Mech, M. Pharr, and P. Prusinkiewicz. Realistic modeling and rendering of plant ecosystems. In Computer Graphics, 32(3), Siggraph 98 Conference Proceedings, pages 275-286, New York, 1998. ACM Press.

[7] W. G. Hopkins. Introduction to Plant Physiology, pages 125-141. John Wiley \& Sons Inc., second edition, 1999.

[8] B. Lear. Autumn leaves. ChemMatters, 10:7-13, 1986.

[9] D. L. MacAdam. Color Measurements Theme and Variations. Springer Verlag, Berlin, Germany, 1981.

[10] R. Mech and P. Prusinkiewicz. Visual models of plants interacting with their environment. In Siggraph 96 Conference Proceedings, pages 397-410, 1996.

[11] S. Mochizuki, D. Cai, T. Komori, H. Kimura, and R. Hori. Virtual autumn coloring system based on biological and fractal model. In Proceedings of the 9th Pacific Conferene on Computer Graphics and Applications, 2001.

[12] J. H. M. Thornley. Mathematical Models in Plant Physiology, pages 42-58, 107-143. Academic Press, 1975. 